\title{
Dielectric Properties of a Once-Broken Rod Polypeptide
}

\author{
Masako FujIwARA and Nobuhiko SAITô \\ Department of Applied Physics, Waseda University, \\ Tokyo 160, Japan.
}

(Received June 15, 1978)

\begin{abstract}
The theoretical interpretation of the dipole moment and the relaxation time measured experimentally on the once-broken poly $(\gamma$-benzyl L-glutamate) molecule is presented in this paper. The rotatory motion of molecule can be separated by using the center-of-mass coordinates and the appropriate variables. The diffusion equation is solved by a perturbation method, and the relaxation time thus obtained can explain the experimental results.
\end{abstract}

KEY WORDS Once-Broken Rod / Dielectric Measurement / Dipole Moment / Relaxation Time / Diffusion Equation / Center of Mass / Hydrodynamic Interaction / Perturbation Method /

Solution properties of a synthesized once-broken rod polypeptide have so far been examined experimentally by Teramoto, et al. ${ }^{1,2}$ A molecule of poly( $\gamma$-benzyl L-glutamate) (PBLG) consists of two $\alpha$-helical polypeptide chains of equal length jointed in a head-to-head fashion by a flexible initiator residue (of a trimethylenediamine). Light scattering, osmotic pressure, viscosity, sedimentation constant and helix-coil transition temperature have been measured for the PBLG samples of the once-broken type (PBG-B) and the results were compared with those of the straight rod of the same molecular weight (PBG-A).

Theoretical studies of the once-broken rod have been done by a number of workers. ${ }^{3-9} \mathrm{Yu}$ and Stockmayer, ${ }^{5}$ in their pioneer work, calculated the intrinsic viscosity $[\eta]$ using the method of Kirkwood $^{6}$ and obtained results which were in pretty good agreement with experiments. Hassager ${ }^{7}$ corrected these results in a free draining case by taking the center of mass at the origin of the coordinate system in the calculation. He expressed the conformation of the molecule in terms of Eulerian angles and the angle $\chi$ between two helical rods as a fourth variable. Wilemsky ${ }^{8}$ also used this coordinate system for calculating the translational diffusion coefficient, the sedimentation constant and the intrinsic viscosity in the case of a fixed $\chi$ in a $\chi$ dependent form and averaged over $\chi$. Taki and Fujita ${ }^{9}$ have recently calculated the in- trinsic viscosity by means of a method developed by Yamakawa and Fujii $^{10}$ for the worm-like chain model.

The samples of once-broken rods hitherto studied experimentally were polydisperse and possibly contaminated with unbroken rods; and therefore experimental data were far from being completely reliable. Teramoto, et al. $^{11}$ carried out the dielectric measurements on almost completely monodisperse samples of once-broken PBLG and made a substantial improvement on the experimental sides.

The dielectric methods give the dipole moment and the relaxation time of the dissolved molecule with their molecular weight dependence, both of which can be related closely to the molecular conformation. The theories referred to above are insufficient for treating dielectric behaviors of the once-broken rod polypeptides. The coordinate systems they adopted are not necessarily convenient for the present use. Wilemsky and others did not take proper account of the flexibility of the molecule. The dynamical treatment accounting for the molecular flexibility was given only by $\mathrm{Yu}$ and Stockmayer, but they failed to use the centerof-mass coordinate system. The present paper takes their point of view and discusses the problem by employing an appropriate coordinate system to explain the experimental results of Teramoto, et al. 


\section{Fujiwara and N. Saito}

The molecule is assumed to be a rigid assembly of beads. In the dielectric relaxation, only rotatory motion of the molecule is effective and the translational motion should be separated off from over all motion by taking the origin of coordinate system at the center of mass. The rotatory diffusion coefficients can be calculated by taking into account the hydrodynamic interaction after the Kirkwood procedure. A diffusion equation is formulated in the presence of the periodic electric field and the intra-molecular dipolar interaction potential, and this equation is different from those of Hassager and others, which are described by their own variables and have difficulty for treating this problem. The relaxation time calculated in this paper about $30 \%$ of that obtained by $\mathrm{Yu}$ and Stockmayer.

The first part of this paper is devoted to the equilibrium dielectric properties of the once-broken rod solution and then the dynamical theory is discussed to explain the experimental relaxation time.

\section{EQUILIBRIUM PROPERTIES}

Teramoto, et al. measured the molecular weight dependence of the dipole moment $\langle p\rangle^{1 / 2}$ for both PBG-A and PBG-B as shown in Figure 1. The

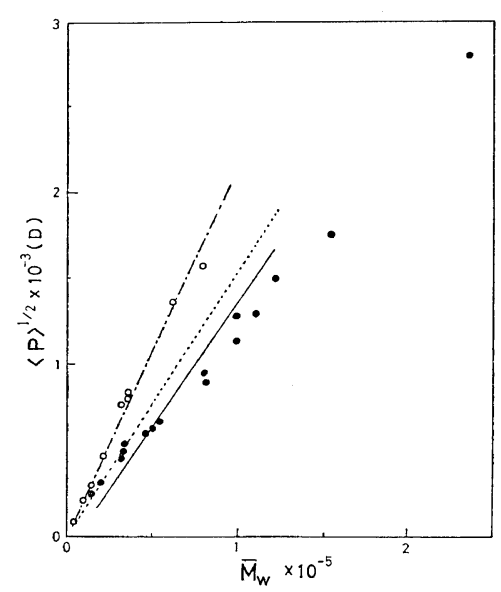

Figure 1. Molecular weight dependence of the average dipole moment: $O$ and $\bullet$ represent the experimental data for PBG-A and PBG-B, respectively. - and --.-- represent the theoretical relations of the simple model for the two molecules; presents our result calculated for PBG-B. dotted-and-dashed line is the theoretical relation between $\langle p\rangle^{1 / 2}$ and the molecular weight for the rigid straight rod and data points for PBG-A follow this line which yields an average value of $4.7 \pm 0.2$ (D) for dipole moment $\mu_{\mathrm{h}}$ per monomeric residue. The dotted line represents the relation expected from a model (dipole moment $\sqrt{2} \mu_{\mathrm{h}} \times$ $(N / 2)$ ) which is composed of two rods of equal length and equal dipole moment $\mu_{\mathrm{h}}(N / 2)$ jointed rigidly at $90^{\circ}$. This model is justified from the consideration that in a weak electric field the orientation of each rod of the once-broken rod molecule is uniform and therefore the average angle between the two rods is $90^{\circ}$, provided that the dipolar interaction between the two rods is ignored. ${ }^{12}$

In the calculations to follow, a PBG-B molecule is assumed to consists of rods of lengths $r$ and $r^{\prime}$ jointed by a flexible initiator in a head-to-head fashion. The average dipole moment of PBG-B is calculated by considering the dipolar interaction $V$ which is approximated as coulomb interaction between the two ends

$$
V=q^{2} / 4 \pi \varepsilon_{0} R
$$

where $R$ is the distance between two $-q$ charges at the ends of the molecule. The coordinates of the two rods are specified by the polar coordinates of the two rods $(r, \theta, \phi)$ and $\left(r^{\prime}, \theta^{\prime}, \phi^{\prime}\right)$ with respect to the cartesian coordinates $(x, y, z)$ with the origin at the joint. The dipole moments of the two rods are

$$
\begin{aligned}
& \boldsymbol{\mu}=q \boldsymbol{r} \\
& \boldsymbol{\mu}^{\prime}=q \boldsymbol{r}^{\prime}
\end{aligned}
$$

respectively. In the presence of the electric field $E$ in the direction of $z$ axis, the equilibrium distribution function of the molecule $F_{0}\left(\theta, \phi, \theta^{\prime}, \phi^{\prime}\right)$ is given, as long as the electric field and $q^{2}$ are small, by

$$
\begin{gathered}
F_{0}\left(\theta, \phi, \theta^{\prime}, \phi^{\prime}\right)=\left(\frac{1}{4 \pi}\right)^{2} \exp \left(\frac{-q^{2}}{4 \pi \varepsilon_{0} k T R}\right) \\
\times \exp \left\{\frac{E}{k T}\left(\mu \cos \theta+\mu^{\prime} \cos \theta^{\prime}\right)\right\}
\end{gathered}
$$

The equilibrium polarization is therefore,

$$
\begin{aligned}
\langle p\rangle= & \int p_{z} F_{0}\left(\theta, \phi, \theta^{\prime}, \phi^{\prime}\right) \mathrm{d} \Omega \mathrm{d} \Omega^{\prime}, \\
\cong & \left(\frac{1}{4 \pi}\right)^{2} \int\left(\mu \cos \theta+\mu^{\prime} \cos \theta^{\prime}\right)\left(1-q^{2} / 4 \pi \varepsilon_{0} k T R\right) \\
& \times\left\{1+\frac{E}{k T}\left(\mu \cos \theta+\mu^{\prime} \cos \theta^{\prime}\right)\right\} \mathrm{d} \Omega \mathrm{d} \Omega^{\prime}
\end{aligned}
$$


where $\mathrm{d} \Omega=\sin \theta \mathrm{d} \theta \mathrm{d} \phi, \mathrm{d} \Omega^{\prime}=\sin \theta^{\prime} \mathrm{d} \theta^{\prime} \mathrm{d} \phi^{\prime}$, and $F_{0}$ is expanded to the first power of the internal potential and the interaction with the external field respectively. $1 / R$ can be expanded as

$$
\begin{aligned}
\frac{1}{R}= & \sum_{n=0}^{N} \frac{r^{n}}{r^{\prime n+1}} \sum_{m=-n}^{n} \frac{(n-m) !}{(n+m) !} \\
& \times P_{n}{ }^{m}(\cos \theta) P_{n}{ }^{m}\left(\cos \theta^{\prime}\right) \cos m\left(\phi-\phi^{\prime}\right) \\
& \left(r^{\prime}>r\right)
\end{aligned}
$$

Substituting this into eq 4 we obtain

$$
\begin{gathered}
\langle p\rangle \cong \frac{E}{(4 \pi)^{2} k T} \int\left(\mu \cos \theta+\mu^{\prime} \cos \theta^{\prime}\right)^{2} \\
\times\left(1-\frac{Q}{R}\right) \mathrm{d} \Omega \mathrm{d} \Omega^{\prime} \\
\cong \frac{E}{3 k T}\left\{\left(\mu^{2}+\mu^{\prime 2}\right)-Q\left[\frac{1}{r^{\prime}}\left(\mu^{2}+\mu^{\prime 2}\right)\right.\right. \\
\left.\left.+\frac{2}{3} \mu \mu^{\prime} \frac{r}{r^{\prime 2}}\right]\right\}
\end{gathered}
$$

where

$$
Q=q^{2} / 4 \pi \varepsilon_{0} k T
$$

for PBG-B molecule we may put $r=r^{\prime}=(N / 2) b$ and $\mu=\mu^{\prime}=(N / 2) \mu_{\mathrm{h}}$;

$$
\left\langle p_{\mathrm{B}}\right\rangle \cong \frac{E}{6 k T}\left(1-\frac{8}{3} \frac{Q}{N b}\right)\left(N \mu_{\mathrm{h}}\right)^{2}
$$

For PBG-A molecule, we may put $\mu=N \mu_{\mathrm{h}}$, $\mu^{\prime}=0$ and $Q=0$,

$$
\left\langle p_{\mathrm{A}}\right\rangle=\frac{E}{3 k T}\left(N \mu_{\mathrm{h}}\right)^{2}
$$

To see the molecular weight dependence of the average dipole moment of the molecule, $\langle p\rangle^{1 / 2}$ is calculated in the two cases mentioned above by using the experimental data; $T=300 \mathrm{~K}, \varepsilon_{0}=10$ and $\mu_{\mathrm{h}}=4.7$ (D). The value of $\left\langle p_{\mathrm{B}}\right\rangle^{1 / 2}$ are represented by the solid line in Figure 1, where the dotted line which represents the simple model mentioned above corresponds to the case $Q=0$ in eq 8 .

The results for $\left\langle p_{B}\right\rangle^{1 / 2}$ agree with the experimental plots in medium molecular weight; for short helical rods, our approximation of coulomb potential is not adequate and for longer ones our treatment for the rigid rod will be insufficient because of the flexibility of the rod.

The average angle $\langle\chi\rangle$ between the two helices for PBG-B molecule is also calculated by defining $\langle\chi\rangle$ through $\cos \langle\chi\rangle=\langle\cos \chi\rangle$,

$$
\begin{aligned}
\langle\cos \chi\rangle= & \int F_{0}\left\{\sin \theta \sin \theta^{\prime} \cos \left(\phi-\phi^{\prime}\right)+\cos \theta \cos \theta^{\prime}\right\} \\
& \times \mathrm{d} \Omega \mathrm{d} \Omega^{\prime}=-\frac{2}{9} \frac{Q}{r} .
\end{aligned}
$$

In this calculation the appropriate equilibrium distribution function in eq 4 is employed. For example, we have

$$
\left.\begin{array}{ll}
\langle\chi\rangle=94.03^{\circ} & \text { for } N=100 \\
\langle\chi\rangle=92.01^{\circ} & \text { for } N=200 \\
\langle\chi\rangle=91.30^{\circ} & \text { for } N=300
\end{array}\right\}
$$

When the degree of polymerization becomes higher, the angle $\langle\chi\rangle$ approaches $90^{\circ}$.

\section{RELAXATION TIME}

The dielectric measurements for PBG-A and PBG-B molecules give the mean relaxation times which have molecular weight $\left(\bar{N}_{\mathrm{w}}\right)$ dependence. Figure 2 shows the logarithmic plots of mean relaxation times vs. $\bar{N}_{\mathrm{w}}$. Here the dotted-anddashed line represents the theoretical relation for rod-like molecule and data points lie on this curve. The relaxation times of $\mathrm{PBG}-\mathrm{B}$ have a molecular weight dependence similar to those of $\mathrm{PBG}-\mathrm{A}$, but they are smaller than those of the latter by a factor of 3 or 4 when compared the same molecular weight. For all samples of PBG-B examined, the relaxation times are larger than those for the PBG-A molecules of half their molecular weight (shown by the broken line in Figure 2). According to the theory of $\mathrm{Yu}$ and Stockmayer, the longest relaxation time of a once-broken rod is half that of a straight rod of equal total length at the limit of infinite length, as is shown by the dotted line in Figure 2. The observed relaxation times of PBG-B are smaller than those of Yu and Stockmayer and larger than the dashed line.

Let the once-broken rod carry $2 n+1$ hydrodynamic elements, each having a friction constant $\zeta$; the elements are evenly spaced at a separation $b$ along each rod-like portion and one of these elements has a universal joint. The total length of the molecule is $2 n b$. Use is made of the same coordinates of the molecule as in the previous section. Then the diffusion equation is described by the four variables $\left(\theta, \phi, \theta^{\prime}, \phi^{\prime}\right)$ which are denoted as $q^{\alpha}(\alpha=1,2,3,4)$, respectively. The diffusion equation in $q^{\alpha}$-space is written as 
M. Fujiwara and N. SaIto

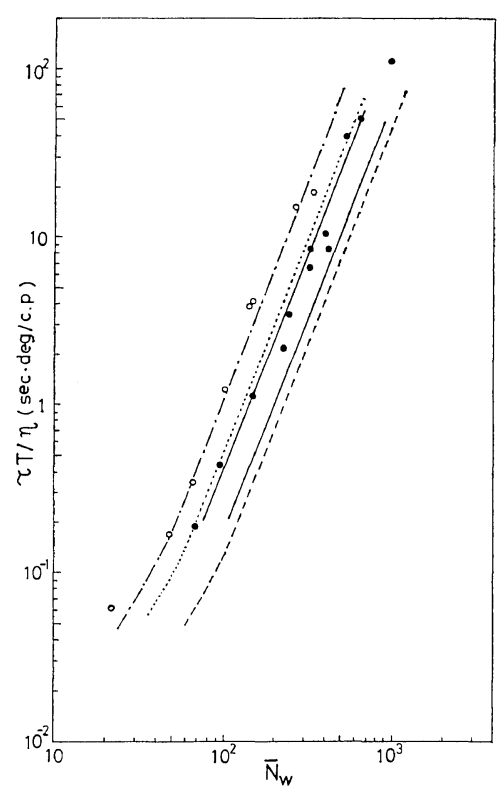

Figure 2. Logarithmic plots of mean relaxation time vs. $\bar{N}_{\mathrm{w}}$ : - - - and - - represent the Perrin's relation for PBG-A and that of half length, respectively. ----- represents the result of Yu and Stockmayer's theory; — represents our theoretical relation; the upper line for the head-to-tail fashioned and the lower for the head-to-head fashioned. The symbols are the same as those in Figure 1.

$$
\begin{aligned}
\frac{\partial f}{\partial t}= & \sum_{\alpha} \sum_{\beta} \sqrt{g} \frac{-1}{\partial \sqrt{g}} \frac{\alpha^{\alpha \beta}}{\partial q^{\beta}}\left(\frac{\partial}{\partial q^{\alpha}}+\frac{1 \partial V}{k T \partial q^{\alpha}}\right. \\
& \left.-\frac{1}{k T} \mu E_{\alpha}\right) f
\end{aligned}
$$

The cartesian coordinates are transformed into $q^{\alpha}$ variables where $g$ is the determinant of the covariant components of the metric tensor. $E$ is the electric field applied along $z$ axis, and the intra-molecular potential $V$ (which is the same as in the previous section) is also considered. The diffusion tensor is given by

$$
D^{\alpha \beta} / k T=g^{\alpha \beta} / \zeta+T^{\alpha \beta}
$$

where $g^{\alpha \beta}$ is the contravariant component of the metric tensor, and $T$ refers to the Oseen tensor describing hydrodynamic interactions between frictional elements.

$\mathrm{Yu}$ and Stockmayer took the coordinate system with the origin at the joint of two rods and did not consider the potential $V$. For the first rod, they obtained;

$$
\begin{aligned}
g^{\theta \theta} & =1 / \sigma_{0}, \\
\sigma_{0} & =\sum_{l=0}^{n}(b l)^{2}=\frac{1}{3} n^{3} b^{2}
\end{aligned}
$$

and

$$
\left.\begin{array}{rl}
D^{\theta \theta} & =\frac{k T}{\sigma_{0} \zeta}\left(1+\gamma_{0}\right) \\
\gamma_{0} & =\frac{\zeta b}{4 \pi \eta \sigma_{0}} \sum_{n \geq l \geq s \geq 0} \sum_{s} \frac{l s}{(l-s)}
\end{array}\right\}
$$

where $\eta$ is the solvent viscosity and for the second rod, $D^{\theta^{\prime} \theta^{\prime}}=D^{\theta \theta} \equiv D_{\mathrm{ys}}^{\theta \theta}$. The relaxation time obtained is

$$
\tau_{\mathrm{ys}}=1 / 2 D_{\mathrm{ys}}^{\theta \theta}
$$

Thus in Yu and Stockmayer's theory, each rodlike portion is assumed to orient independently. $D_{\mathrm{ys}}^{\theta \theta}\left(\tau_{\mathrm{ys}}\right)$ is just twice (one half) that of the PBG-A molecule.

The relaxation time calculated by $\mathrm{Yu}$ and Stockmayer includes the effect of the translational motion, so that it is conceivable that $\tau_{\text {ys }}$ is larger than the experimental value. From this consideration, we are lead to estimate the rotatory relaxation time by separating the translational motion from the overall molecular motion by taking the center of mass as the origin of the coordinate system. In the course of this study, ${ }^{13}$ we noticed the paper by Hassager, Wilemsky, and others mentioned in the Introduction. They took the center of mass as the origin, but their coordinates are not necessarily adequate for treating the dielectric properties.

In our coordinate system, the position vector $\boldsymbol{R}_{i}$ of the $i$-th element is given as,

$$
\begin{aligned}
\boldsymbol{R}_{i} & \equiv \boldsymbol{r}_{i}-\boldsymbol{r}_{\mathrm{cm}} \\
& =b\left\{(i-(n / 4)) \sin \theta \cos \phi-(n / 4) \sin \theta^{\prime} \cos \phi^{\prime}\right\} \boldsymbol{e}_{x} \\
& +b\left\{(i-(n / 4)) \sin \theta \sin \phi-(n / 4) \sin \theta^{\prime} \sin \phi^{\prime}\right\} \boldsymbol{e}_{y} \\
& +b\left\{(i-(n / 4)) \cos \theta-(n / 4) \cos \theta^{\prime}\right\} \boldsymbol{e}_{z}
\end{aligned}
$$

where $\boldsymbol{r}_{i}$ and $\boldsymbol{r}_{\mathrm{cm}}$ are respectively the vectors of the $i$-th element and the center of mass of the molecule from the joint, and $\boldsymbol{e}_{x}, \boldsymbol{e}_{y}, \boldsymbol{e}_{z}$ are the unit vectors of the $(x, y, z)$ coordinates.

By making use of this coordinate system, we can calculate the covariant components of the metric tensor, as for instance,

$g_{\theta \theta}=\sum_{i}^{\operatorname{rod} 1} \frac{\partial \boldsymbol{R}_{i}}{\partial \theta} \cdot \frac{\partial \boldsymbol{R}_{i}}{\partial \theta}+\sum_{j}^{\operatorname{rod} 2} \frac{\partial \boldsymbol{R}_{j}}{\partial \theta} \cdot \frac{\partial \boldsymbol{R}_{j}}{\partial \theta}=\frac{5}{24} n^{3} b^{2} \equiv \sigma$ 
Dielectric Properties of a Once-Broken Rod

and

$$
\begin{aligned}
g_{\theta \theta^{\prime}} & =\sum_{i}^{\operatorname{rod} 1} \frac{\partial \boldsymbol{R}_{i}}{\partial \theta} \cdot \frac{\partial \boldsymbol{R}_{i}}{\partial \theta}+\sum_{j}^{\operatorname{rod} 2} \frac{\partial \boldsymbol{R}_{j}}{\partial \theta} \cdot \frac{\partial \boldsymbol{R}_{j}}{\partial \theta} \\
& =-\frac{3}{5} \sigma\left\{\cos \theta \cos \theta^{\prime} \cos \left(\phi-\phi^{\prime}\right)+\sin \theta \sin \theta^{\prime}\right\}
\end{aligned}
$$

Thus $g_{\alpha \beta}$ 's are the functions of four variables and are not diagonal, contrary to the results of $\mathrm{Yu}$ and Stockmayer which state that $g_{\theta \theta}^{\mathrm{ys}}=\sigma_{0}=n^{3} b^{2} / 3$ and $g_{\theta^{\prime}}^{\mathrm{yS}}=0$. It is noticed that the value of $g_{\theta \theta}$, or $\sigma$ is smaller than $g_{\theta \theta}^{\mathrm{ys}}$ or $\sigma_{0}$. Further we have

$$
g=\left|g_{\alpha \beta}\right|=(4 / 5)^{2}\left\{1-(3 / 5)^{2} \cos ^{2} \chi\right\} \sin ^{2} \theta \sin ^{2} \theta^{\prime} \sigma^{4}
$$

and

$$
g^{-1}=(5 / 4)^{2}\left\{1+(3 / 5)^{2} \cos ^{2} \chi\right\} \sin ^{-2} \theta \sin ^{-2} \theta^{\prime} \sigma^{-4}
$$

The contravariant components, $g^{\mu \beta}$, are also calculated. In particular,

$$
\begin{aligned}
& g^{\theta \theta} \cong(\left.\frac{5}{4}\right)^{2} \frac{1}{\sigma}\left[1-\left(\frac{3}{5}\right)^{2}\left\{\cos ^{2}\left(\phi-\phi^{\prime}\right)\right.\right. \\
&\left.\left.+\cos ^{2} \theta^{\prime} \sin ^{2}\left(\phi-\phi^{\prime}\right)-\cos ^{2} \chi\right\}\right] \\
&= \frac{1}{\sigma}\left[1+\left(\frac{3}{4}\right)^{2}\left\{\sin ^{2} \theta^{\prime} \sin ^{2}\left(\phi-\phi^{\prime}\right)+\cos ^{2} \chi\right\}\right] \\
& g^{\theta \theta \prime} \cong\left(\frac{3}{5}\right)\left(\frac{5}{4}\right)^{2} \frac{1}{\sigma}\left[\left\{\cos \theta \cos \theta^{\prime} \cos \left(\phi-\phi^{\prime}\right)\right.\right. \\
&\left.\quad+\sin \theta \sin \theta^{\prime}\right\}\left\{1+\left(\frac{3}{5}\right)^{2} \cos { }^{2} \chi\right\} \\
&\left.\quad-\left(\frac{3}{5}\right)^{2} \cos \chi \cos \left(\phi-\phi^{\prime}\right)\right] \\
& g^{\theta \phi^{\prime} \cong}\left(\frac{3}{5}\right)\left(\frac{5}{4}\right)^{2} \frac{1}{\sigma} \sin \left(\phi-\phi^{\prime}\right)\left\{-\cos \theta+\left(\frac{3}{5}\right)^{2}\right. \\
&\left.\times\left(\cos ^{2} \chi \cos \theta+\cos \chi \cos \theta^{\prime}\right)\right\}
\end{aligned}
$$

where the terms with powers higher than $(3 / 5)^{3}$ are ignored. Next, we must calculate the hydrodynamic interactions in eq 13 ; this is more complicated because $g^{\alpha \beta}$ is not diagonal. The $\alpha \beta$ component of the Oseen tensor is given by

$$
\begin{aligned}
T^{\alpha \beta}= & \sum_{l, s} \sum_{s} \frac{1}{8 \pi \eta R_{l s}} \sum_{\sigma} \sum_{\nu} g^{\alpha \sigma} g^{\beta \nu}\left[\frac{\partial \boldsymbol{R}_{l}}{\partial q^{\sigma}} \cdot \frac{\partial \boldsymbol{R}_{s}}{\partial q^{\nu}}\right. \\
& \left.+\left(\boldsymbol{R}_{l s} \frac{\partial \boldsymbol{R}_{l}}{\partial q^{\sigma}}\right)\left(\boldsymbol{R}_{l s} \frac{\partial \boldsymbol{R}_{s}}{\partial q^{\nu}}\right) / \boldsymbol{R}_{l s}^{2}\right]
\end{aligned}
$$

where $\boldsymbol{R}_{l s}$ is the distance between the elements $l$ and $s$. The detailed calculations are given in Appendix I. The diffusion coefficient $D^{\theta \theta}$ is thus obtained by substituting $T^{\theta \theta}$ into eq 13 .

$$
D^{\theta \theta} \mid k T \cong\left(g^{\theta \theta} / \zeta\right)\left\{1+\frac{3}{8} b^{2} g^{\theta \theta} \times \frac{5}{12} n^{3}(\ln n-1)\right\}
$$

where we may put $\zeta=3 \pi \eta b$, which is obtained assuming that an $\alpha$-helical polypeptide is approximated by a linear array of beads of diameter $b$ with separation distance $b$. Further approximation is made that $g^{\theta \theta} \cong(1 / \sigma)$ in the parenthesis of the second factor, and so we have

$$
\begin{gathered}
g^{\theta \theta} \cong \frac{8}{5} g_{\text {ys }}^{\theta \theta} \\
D^{\theta \theta} / k T \cong\left(g^{\theta \theta} / 5\right)\left\{1+\frac{3}{8} b^{2} g_{\mathrm{ys}}^{\theta \theta} \times \frac{2}{3} n^{3}(\ln n-1)\right\} \\
\cong\left(g^{\theta \theta} / \zeta\right)\left(1+\gamma_{0}\right)
\end{gathered}
$$

The second factor $\left(1+\gamma_{0}\right)$ is equal to that of $\mathrm{Yu}$ and Stockmayer. In the same way,

$$
D^{\phi \dot{\phi}} / k T \cong g^{\phi \dot{\phi}}\left(1+\gamma_{0}\right) / \zeta
$$

Other coefficients can be obtained by more tedious calculation, but explicit expressions are not given here.

The diffusion operator $L$ in the first term of the rhs of eq 12 can then be written as the sum of two terms, by putting $D \equiv D^{\theta \theta}$,

$$
L \equiv \frac{1}{D} \sum_{\alpha} \sum_{\beta} \frac{1}{\sqrt{g}} \frac{\partial \sqrt{g}}{\partial q^{\beta}} D^{\alpha \beta} \frac{\partial}{\partial q^{\alpha}}=L_{0}+L^{\prime}
$$

where

$$
\begin{gathered}
L_{0}=\nabla_{\theta \dot{\phi}}^{2}+\nabla_{\theta^{\prime} \phi^{\prime}}^{2} \\
\nabla_{\theta \dot{\phi}}^{2}=\frac{1}{\sin \theta} \frac{\partial}{\partial \theta} \sin \theta \frac{\partial}{\partial \theta}+\frac{1}{\sin ^{2} \theta} \frac{\partial^{2}}{\partial \phi^{2}}
\end{gathered}
$$

and

$$
\begin{aligned}
L^{\prime}= & \frac{1}{2}\left(\frac{3}{5}\right)^{2} \cos ^{2} \chi\left(\nabla_{\theta \phi}^{2}+\nabla_{\theta^{\prime} \phi^{\prime}}^{2}\right)+\left(\frac{3}{4}\right)^{2}\left(\hat{F}_{1}+\hat{F}_{2}\right) \\
& +\frac{1}{D} \sum_{\alpha \neq \beta} \frac{1}{\sqrt{g}} \frac{\partial \sqrt{g}}{\partial q^{\alpha}} D^{\alpha \beta} \frac{\partial}{\partial q^{\beta}} \\
\hat{F}_{1} & =\frac{1}{\sin \theta} \frac{\partial}{\partial \theta} \sin \theta G_{1} \frac{\partial}{\partial \theta}+\frac{1}{\sin ^{2} \theta} \frac{\partial}{\partial \phi} G_{1} \frac{\partial}{\partial \phi} \\
\hat{F}_{2} & =\frac{1}{\sin \theta^{\prime}} \frac{\partial}{\partial \theta^{\prime}} \sin \theta^{\prime} G_{2} \frac{\partial}{\partial \theta^{\prime}}+\frac{1}{\sin ^{2} \theta^{\prime}} \frac{\partial}{\partial \phi^{\prime}} G_{2} \frac{\partial}{\partial \phi^{\prime}} \\
G_{1} & =\sin ^{2} \theta^{\prime} \sin ^{2}\left(\phi-\phi^{\prime}\right)+\cos ^{2} \chi \\
G_{2} & =\sin ^{2} \theta \sin ^{2}\left(\phi-\phi^{\prime}\right)+\cos ^{2} \chi
\end{aligned}
$$


Here we have used eq 20, 21, and 22, and the last term in eq 32 indicates the summation over offdiagonal terms.

Equation 12 can be written as

$$
\begin{gathered}
-\frac{1}{D} \frac{\partial f}{\partial t}=\left(L_{0}+L^{\prime}+W-\hat{E}\right) f \\
W=\frac{1}{D} \sum_{\alpha} \sum_{\beta} \frac{1}{\sqrt{g}} \frac{\partial \sqrt{g}}{\partial q^{\alpha}} \frac{D^{\alpha \beta}}{k T} \frac{\partial V}{\partial q^{\beta}} \\
\hat{E}=\frac{1}{D} \sum_{\alpha} \sum_{\beta} \frac{1}{\sqrt{g}} \frac{\partial \sqrt{g}}{\partial q^{\alpha}} \frac{D^{\alpha \beta}}{k T} \mu E_{\beta}
\end{gathered}
$$

The usual technique for solving this equation, by regarding $\hat{E}$ as a perturbation, gives, for the solution periodic in time,

$$
\begin{aligned}
& f \cong f_{0}+E \mathrm{e}^{i \omega t} f_{1}\left(\theta, \phi, \theta^{\prime}, \phi^{\prime}\right) \\
& f_{0}=\left(\frac{1}{4 \pi}\right)^{2} \exp \left(-q^{2} / 4 \pi \varepsilon_{0} R k T\right) \\
& f_{1}=\frac{b_{1}}{i \omega / D+\lambda_{1}} \phi_{1}^{(0)}+\frac{b_{2}}{i \omega / D+\lambda_{2}} \phi_{2}^{(0)}
\end{aligned}
$$

where $\phi_{j}^{(0)}$ is the $j$-th eigenfunction of $L_{0}$, in particular

$$
\begin{aligned}
{\phi_{1}}^{(0)} & =\frac{\sqrt{6}}{8 \pi}\left(\cos \theta+\cos \theta^{\prime}\right) \\
\phi_{2}{ }^{(0)} & =\frac{\sqrt{6}}{8 \pi}\left(\cos \theta-\cos \theta^{\prime}\right)
\end{aligned}
$$

and

$$
\begin{aligned}
b_{j}= & \frac{1}{E D} \int \phi_{j}^{\left({ }^{(0)}\right.} \sum_{\alpha, \beta} \sqrt{g^{-1}} \frac{\partial \sqrt{g}}{\partial q^{\beta}} \\
& \times\left\{\left(D^{\alpha \beta} / k T\right) \mu E_{\alpha} f_{0}\right\} \mathrm{d} \Omega \mathrm{d} \Omega^{\prime}
\end{aligned}
$$

The eigenvalues $\lambda_{1}$ and $\lambda_{2}$ of the operator $L_{0}+L^{\prime}$ $+W$ are given by

$$
\begin{aligned}
& \lambda_{1} \cong \lambda_{0}-\left(A_{1}+A_{2}+B_{1}+B_{2}\right) \\
& \lambda_{2} \cong \lambda_{0}-\left(A_{1}-A_{2}+B_{1}-B_{2}\right)
\end{aligned}
$$

The detailed calculations of $A, B$ and $b_{j}$ are given in Appendices II and III. We put $\mu=\mu^{\prime}\left(b_{2}=\right.$ $\left.b_{2}{ }^{\prime}\left(\mu-\mu^{\prime}\right)=0\right)$, for the PBG-B molecule synthesized in a head-to-head fashion with arms of equal length, and we have

$$
\begin{aligned}
f_{1} \cong \frac{\sqrt{6}}{24 \pi k T}(3.73 & \left.-\frac{13}{4} \frac{Q}{r}\right) \frac{\left(\mu+\mu^{\prime}\right)}{i \omega / D+\lambda_{1}} \\
& \times\left(\cos \theta+\cos \theta^{\prime}\right)
\end{aligned}
$$

Therefore the necessary eigenvalue is only $\lambda_{1}$,

$$
\lambda_{1} \cong 3.73+1.62 \frac{Q}{r} \cong 3.98
$$

where $Q$ is the same as eq 7. The relaxation time is given in comparison with that of $\mathrm{Yu}$ and Stockmayer,

$$
\tau=\frac{1}{\lambda_{1} D} \cong 0.314 \tau_{\mathrm{ys}}
$$

where eqs 16 and 27 are combined. This is a much smaller value than $\tau_{\mathrm{ys}}$. To see the effects of the perturbations, the relaxation time $\tau^{\prime}$ without the introduction of the intramolecular potential $W$ can be obtained as

$$
\tau^{\prime} \cong 0.335 \tau_{\text {ys }}
$$

This shows that the effect of the center-of-mass coordinate system is essentially important. The experimental relaxation times seem more or less larger than those calculated here; this may be regarded as an effect of the large initiator residue of the joint which hinders the free rotation of the two rods in the real PBG-B molecule.

The polarization is given by

$$
\begin{aligned}
\langle p(t)\rangle & =\int\left(\mu \cos \theta+\mu^{\prime} \cos \theta^{\prime}\right) f_{1} E \mathrm{e}^{i \omega t} \mathrm{~d} \Omega \mathrm{d} \Omega^{\prime} \\
& \cong \frac{2 \mu^{2}}{3 k T}\left(1-\frac{49}{37} \frac{Q}{r}\right) \frac{E \mathrm{e}^{i \omega t}}{1+i \omega \tau}
\end{aligned}
$$

This value is close to that of eq 8 when $\omega$ is put to 0 . $((49 / 37) \times 2 \cong(8 / 3))$. The slight discrepancy comes from the approximate calculation in deriving eq 48 , while the value of eq 8 can be considered exact in the case $\omega=0$.

If the PGB-B molecule is considered as headto-tail, we may put $\mu^{\prime}=-\mu$ in eq 38 and the eigenvalue $\lambda_{2}$, the relaxation time $\tau^{\prime \prime}$ is given as

$$
\begin{aligned}
& \tau^{\prime \prime}=\frac{1}{\lambda_{2} D} \cong 0.858 \tau_{\text {ys }} \\
& \lambda_{2} \cong 1.23-1.45 \frac{Q}{r} \cong 1.46
\end{aligned}
$$

The two relaxation times $\tau$ and $\tau^{\prime \prime}$ are represented as full lines in Figure 2, the upper is $\tau^{\prime \prime}$ and the lower is $\tau$. The dynamical behavior is found to be different between the two forms of the once-broken rod molecule. This was not discussed in $\mathrm{Yu}$ and Stockmayer's theory.

\section{APPENDIX I}

\section{Calculation of $T^{\alpha \beta}$}

The terms $l$ and $s$ in eq 25 on the different rods 
are dropped for their fractional error of $O\left(n^{-1}\right)$, so that $\boldsymbol{R}_{l s}$ is the vector on the either rod. There are some relationships as to the first term, $\left(\partial \boldsymbol{R}_{l} / \partial \theta\right)$. $\left(\partial \boldsymbol{R}_{\boldsymbol{s}} / \partial \phi\right)=0$ and $\left(\partial \boldsymbol{R}_{l} / \partial \theta^{\prime}\right) \cdot\left(\partial \boldsymbol{R}_{\boldsymbol{g}} / \partial \boldsymbol{\phi}^{\prime}\right)=0$; as to the second term, $\left(\boldsymbol{R}_{l s}\left(\partial \boldsymbol{R}_{l} / \partial q^{\alpha}\right)\right)\left(\boldsymbol{R}_{l s}\left(\partial \boldsymbol{R}_{s} / \partial q^{\beta}\right)\right)=0$ when $q^{\alpha}=(\theta, \phi)$ and $q^{\beta}=\left(\theta^{\prime}, \phi^{\prime}\right)$ respectively. The terms $g^{\alpha \sigma} g^{\beta \nu}$ are of the order of $(3 / 5)^{2}$ when $\alpha, \beta=$ $(\theta, \phi)$ and $\sigma, \nu=\left(\theta^{\prime}, \phi^{\prime}\right)$, and can be ignored. Thus we obtain

$$
\begin{aligned}
& \frac{8 \pi \eta}{b} T^{\theta \theta}=\left(g^{\theta \theta}\right)^{2}\left[\sum_{l, s}^{\text {rod } 1} \frac{(l-(n / 4))(s-(n / 4))}{|l-s|}\right. \\
& \quad+\sum_{l, s}^{\text {rod } 2} \frac{(-n / 4)^{2}}{|l-s|}+\sum_{l, s}^{\operatorname{rod} 2} \frac{(-n / 4)^{2}}{|l-s|} \\
& \left.\quad \times\left\{\sin \theta^{\prime} \cos \theta \cos \left(\phi-\phi^{\prime}\right)-\sin \theta \cos \theta^{\prime}\right\}^{2}\right] \\
& \quad+2 g^{\theta \theta} g^{\theta \prime^{\prime}} \sum_{l, s}^{\operatorname{rod} 1} \frac{(-n / 4)(l-(n / 4))}{|l-s|} \\
& \quad \times\left\{\cos \theta \cos \theta^{\prime} \cos \left(\phi-\phi^{\prime}\right)+\sin \theta \sin \theta^{\prime}\right\} \\
& \quad+2 g^{\theta \theta} g^{\phi \prime^{\prime}} \sum_{l, s}^{\operatorname{rod} 1} \frac{(-n / 4)(l-(n / 4))}{|l-s|} \\
& \quad \times \cos \theta \sin \theta \sin \left(\phi-\phi^{\prime}\right)
\end{aligned}
$$

Substituting the values of $g^{\theta \theta}, g^{\theta \theta^{\prime}}, g^{\theta \phi^{\prime}}$ the summations are calculated for sufficiently large $n$,

$$
\begin{aligned}
\frac{8 \pi \eta}{b} & T^{\theta \theta}=g^{\theta \theta} n^{3}(\ln n-1)\left\{\frac{5}{12} g^{\theta \theta}+\mathscr{F} / \sigma\right\} \\
\mathscr{F}= & \frac{7}{100}\left\{-1+\frac{9}{14} \sin ^{2} \theta \sin ^{2}\left(\phi-\phi^{\prime}\right)\right\} \\
& \times\left\{\sin \theta^{\prime} \cos \theta \cos \left(\phi-\phi^{\prime}\right)-\sin \theta \cos \theta^{\prime}\right\}^{2}
\end{aligned}
$$

Here the term $\mathscr{F} / \sigma$ can be neglected and we have

$$
T^{\theta \theta} \cong \frac{b}{8 \pi \eta}\left(g^{\theta \theta}\right)_{2} \frac{5}{12} n^{3}(\ln n-1)
$$

$T^{\phi \dot{\phi} \phi}$ is also calculated as

$$
T^{\phi \phi}=\frac{1}{\sin ^{2} \theta} T^{\theta \theta}
$$

under the same approximation.

\section{APPENDIX II}

Calculation of $A_{1}, A_{2}, B_{1}$, and $B_{2}$

$A_{1}$ and $A_{2}$ are the contributions to the eigenvalue from the perturbation $L^{\prime}$, and $B_{1}$ and $B_{2}$ are those from $W$.

$$
\begin{aligned}
A_{1}= & C^{2} \int \cos \theta\left\{\frac{1}{2}\left(\frac{3}{5}\right)^{2} \cos ^{2} \chi \frac{1}{\sin \theta} \frac{\partial \sin \theta}{\partial \theta} \frac{\partial}{\partial \theta}\right. \\
& \left.+\left(\frac{3}{4}\right)^{2} \frac{1}{\sin \theta} \frac{\partial \sin \theta}{\partial \theta} G_{1} \frac{\partial}{\partial \theta}\right\} \cos \theta \mathrm{d} \Omega \mathrm{d} \Omega^{\prime}
\end{aligned}
$$

where

$$
C=\sqrt{3} / 4 \pi
$$

and $G_{1}$ is given by eq 33. $A_{2}$ is the effect from the off-diagonal terms of the diffusion operator:

$$
A_{2}=\frac{C^{2}}{D} \int \cos \theta\left(\frac{1}{\sin \theta} \frac{\partial \sin \theta}{\partial \theta} D^{\theta \theta^{\prime}} \frac{\partial}{\partial \theta^{\prime}}\right) \cos \theta^{\prime} \mathrm{d} \Omega \mathrm{d} \Omega^{\prime}
$$

Similary,

$$
\begin{aligned}
& B_{1}=\frac{C^{2}}{D k T} \int \cos \theta\left(\frac{1}{\sin \theta} \frac{\partial \sin \theta}{\partial \theta} D^{\theta \theta^{\prime}} \frac{\partial V}{\partial \theta^{\prime}}\right) \cos \theta \mathrm{d} \Omega \mathrm{d} \Omega^{\prime} \\
& B_{2}=\frac{C^{2}}{k T} \int \cos \theta\left(\nabla_{\theta, \varphi}^{2}+\nabla_{\theta^{\prime}, \varphi^{\prime}}^{2}\right) V \cos \theta^{\prime} \mathrm{d} \Omega \mathrm{d} \Omega^{\prime}
\end{aligned}
$$

The intramolecular potential $V$ is the same as eq 1 and use is made of the expansion of eq 5 for $(1 / R)$. It is noted that $A_{2}$ and $B_{1}$ are the diagonal elements of the secular equation and $A_{2}$ and $B_{2}$ are the off-diagonal elements; the latter comes out to have the opposite sign in the eigenvalue $\lambda_{2}$ contrary to in $\lambda_{1}$. The results are as follows.

$$
\left.\begin{array}{l}
A_{1}=-0.48 \\
A_{2}=-1.25 \\
B_{1}=-0.08(Q / r) \\
B_{2}=-1.53(Q / r)
\end{array}\right\}
$$

where $Q$ is given in eq 7 .

\section{APPENDIX III}

Calculation of $b_{j}$

In eq $35, E$ is in $z$-direction so that,

$$
\begin{aligned}
& E_{\phi}=E_{\phi^{\prime}}=0 \\
& E_{\theta}=-E \sin \theta, \quad E_{\theta^{\prime}}=-E \sin \theta^{\prime} .
\end{aligned}
$$

Since $E$ is assumed to be small, we calculate the operator $\hat{E}$ noting that the terms orthogonal to $\cos \theta$ and $\cos \theta^{\prime}$ are dropped in eq 41 . 


$$
\begin{aligned}
b_{1}= & \frac{C}{\sqrt{2} D k T} \int\left(\cos \theta+\cos \theta^{\prime}\right) \sum_{\alpha, \beta}^{\theta, \theta^{\prime}}\left(\frac{1}{\sqrt{g}} \frac{\partial \sqrt{g}}{\partial q^{\alpha}}\right. \\
& \left.\times D^{\theta \alpha} \mu \sin \theta+\frac{1}{\sqrt{g}} \frac{\partial \sqrt{g}}{\partial q^{\beta}} D^{\theta^{\prime} \beta} \mu^{\prime} \sin \theta^{\prime}\right) \\
& \times\left(\frac{1}{4 \pi}\right)^{2}(1-V / k T) \mathrm{d} \Omega \mathrm{d} \Omega^{\prime} \\
= & \frac{C}{4 \sqrt{2} D k T}\left(\lambda_{0}-A_{1}-A_{2}-\frac{13}{4} \frac{Q}{r}\right)\left(\mu+\mu^{\prime}\right) \\
= & b_{1}{ }^{\prime}\left(\mu+\mu^{\prime}\right)
\end{aligned}
$$

where

$$
b_{1}{ }^{\prime}=\frac{C}{4 \sqrt{2 D k T}}\left(\lambda_{0}-A_{1}-A_{2}-\frac{13}{4} \frac{Q}{r}\right)
$$

and $C$ is the same as eq II.2. $\lambda_{0}$ is the nonperturbed eigenvalue and $A_{1}, A_{2}$ are given in the previous Appendix. Similarly we get

$$
b_{2}=b_{2}{ }^{\prime}\left(\mu-\mu^{\prime}\right)
$$

where

$$
b_{2}^{\prime}=\frac{C}{4 \sqrt{2} D k T}\left(\lambda_{0}-A_{1}-A_{2}-\frac{2}{3} \frac{Q}{r}\right)
$$

\section{REFERENCES}

1. A. Teramoto, T. Yamashita, and H. Fujita, $J$. Chem. Phys., 46, 1919 (1967).

2. K. Nakagawa, N. Nishioka, A. Teramoto, and $\mathbf{H}$. Fujita, Polym. J., 4, 332 (1973).

3. R. Pecora, Macromolecules, 2,31 (1969).

4. J.E. Hearst and W.H. Stockmayer, J. Chem. Phys., 37, 1425 (1962).

5. H. Yu and W. H. Stockmayer, J. Chem. Phys., 47, 1369 (1967).

7. O. Hassager, J. Chem. Phys., 60, 2111 (1974).

6. J.G. Kirkwood, Rectrav. Chim., 68, 649(1949).

8. G. Wilemsky, Macromolecules, 10, 28 (1977).

9. N. Taki and H. Fujita, Polym. J., 7, 637 (1975).

10. H. Yamakawa and M. Fujii, Macromolecules, 7, 128 (1974).

11. T. Matsumoto, N. Nishioka, A. Teramoto, and H. Fujita, Macromolecules, 7, 824 (1974).

12. H. Kihara, K. Tanno, and A. Wada, Polym. J., 5, 324 (1973).

13. M. Fujiwara and N. Saito, Polym. J., 9, 625 (1977). The center-of-mass coordinate system was used in the dynamics of bending in the helix-coil transition. 